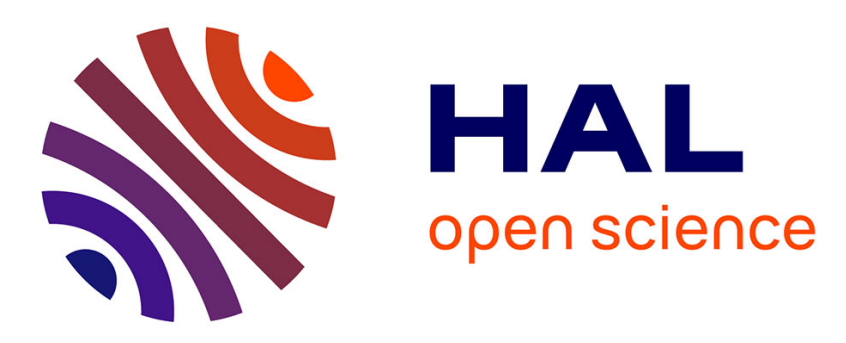

\title{
Cellulose-binding domains: cellulose associated-defensive sensing partners?
}

\author{
Bernard Dumas, Arnaud Bottin, Elodie Gaulin, Marie-Thérèse
}

Esquerré-Tugayé

\section{- To cite this version:}

Bernard Dumas, Arnaud Bottin, Elodie Gaulin, Marie-Thérèse Esquerré-Tugayé. Cellulose-binding domains: cellulose associated-defensive sensing partners?. Trends in Plant Science, 2008, 13 (4), pp.160-4. 10.1016/j.tplants.2008.02.004 . hal-00320691

\section{HAL Id: hal-00320691 https://hal.science/hal-00320691}

Submitted on 11 Sep 2008

HAL is a multi-disciplinary open access archive for the deposit and dissemination of scientific research documents, whether they are published or not. The documents may come from teaching and research institutions in France or abroad, or from public or private research centers.
L'archive ouverte pluridisciplinaire HAL, est destinée au dépôt et à la diffusion de documents scientifiques de niveau recherche, publiés ou non, émanant des établissements d'enseignement et de recherche français ou étrangers, des laboratoires publics ou privés. 
Dumas et al.

1 Cellulose Binding Domains: cellulose-associated defense sensing $2 \quad$ partners?

3

4

5

6

7

8 Bernard Dumas*, Arnaud Bottin, Elodie Gaulin and Marie-Thérèse Esquerré-Tugayé*

9

10 UMR 5546 CNRS-Université Paul Sabatier Toulouse III, Pôle de Biotechnologie

11 Végétale, 24 Chemin de Borde-Rouge, BP42617 Auzeville, 31326 Castanet-Tolosan,

12 France

13

14 *Corresponding authors: Dumas, B. (dumas@scsv.ups-tlse.fr), Esquerré-Tugayé, M.T.

15 (esquerre@scsv.ups-tlse.fr) 


\section{Dumas et al.}

\section{Abstract}

18 The cellulose-binding domains (CBDs) of CBEL, the cellulose-binding elicitor lectin of

19 Phytophthora, are potent elicitors of plant defense responses. Induction of defense has also

20 been reported in various cellulose-deficient mutants of Arabidopsis thaliana. Based on these

21 observations, we propose a model linking cellulose alteration to defense induction. This

22 integrates the fast increase in cytosolic calcium recorded in response to CBEL, mechano-

23 stimulated calcium uptake mechanisms, and proteins that interact functionally with the

24 cellulose synthase complex. In this context, CBDs emerge as new tools to decipher the

25 signalling cascades that result from cell wall-cellulose perturbations. 


\section{Dumas et al.}

\section{Introduction}

27 Owing to their strategic location surrounding the cell, plant cell walls play an important role

28 in the outcome of plant-microorganism interactions. Understanding of the dialogue that takes

29 place at this interface has progressed as a result of the improved biochemical and molecular

30 knowledge of this cellular compartment. After the first report that plant cell walls contain

31 proteins [1], the concept of the cell wall as a dynamic structure involved in signalling and

32 defense has emerged, as notably illustrated by the ability of pectin-derived oligosaccharides to

33 elicit defense responses [2], and by cell wall reinforcement by structural components

34 following pathogen attack [3,4]. Perturbing the cell wall integrity by mechanical stress such

35 as wounding often induces similar signalling and strengthening effects, thereby suggesting

36 that the cell wall harbours its own surveillance system [5]. With the sequencing of a few plant

37 genomes, particularly of Arabidopsis thaliana, the study of plant cell walls has now entered

38 the genomic era, shedding new light on functional aspects of this important cell compartment.

39 Several reviews of current knowledge about cellulose [6,7], and cell wall sugars and proteins

40 as related to stress, have recently appeared $[8,9]$.

41 Our interest in cellulose as a possible partner of the surveillance system has arisen from

42 several reports linking cellulose to plant defense through the study of Arabidopsis cell wall

43 mutants and microbial effectors. Thus, several mutants having defects in cellulose synthesis

44 were shown to be more resistant to various pathogens $[8,9]$. The possibility that cellulose

45 might be part of a sensing machinery was further supported by the finding that the cellulose-

46 binding domains (CBDs) of the CBEL effector of Phytophthora parasitica are sufficient to

47 elicit plant defense [10]. It is unlikely that this effect implies the enzymatic release of oligo- $\beta$ -

48 glucan elicitors from cellulose because CBEL and more generally CBDs are devoid of

49 hydrolase activity. The presence of CBEL at the mycelium cell surface allows Phytophothora

50 to adhere to cellulosic substrates [11]. 


\section{Dumas et al.}

51 Since altering as well as touching cellulose induce plant defense, one might wonder whether there is any crosstalk between the mechanisms underlying the response to these two different 53 stresses. Hence this article focuses on the emerging links between the cellulose

54 biosynthesizing machinery, plant defense, and CBDs as possible interactants.

\section{Proteins associated with cellulose}

Before reporting the few examples linking cellulose to defense sensing, we will give an overview of the genes and proteins that interact directly or indirectly with cellulose at the biosynthesis and structural levels.

60

\section{Proteins of the cellulose biosynthesizing machinery.}

62 The cell wall cellulose microfibrils consist of several chains of 1,4-linked $\beta$-D-glucose residues tightly assembled by hydrogen bonds. As a major metabolic, energy-demanding

64 process in plants, the biosynthesis of cellulose must be tightly programmed and linked to a source of glucose monomers. The level of cellulose in the cell wall depends primarily on the activity of the cellulose synthase complex. After the discovery of the first cellulose synthase gene (CESA) encoding the catalytic subunit of the complex in cotton (Gossypium hirsutum)

68 [12], genome sequencing data revealed that a large set of CESA genes, comprising at least 10 69 members, is present in the genome of Arabidopsis, and of other plants. CESA proteins are

70 located within the plasma membrane, and share common structural features, notably 71 conserved and more variable domains, and the D,D,D,QXXRW motif for substrate binding 72 and catalysis. The phosphorylation status of cellulose synthase seems to be important for its 73 activity [6].

74 In Arabidopsis, mutant complementation analysis has shown that AtCESA1, AtCESA2, 75 AtCESA3, and AtCESA6 are involved in cellulose biosynthesis of the primary cell wall 


\section{Dumas et al.}

$76[6,7,13,14]$, whereas AtCESA4, AtCESA7 and AtCESA8 are expressed during secondary cell wall formation $[6,7]$. A number of cesA mutants, with mutations scattered along the whole sequence of these various genes, are available. They exhibit growth defects and a decrease in the cellulose content of the cell wall which is accompanied in a few cases by deposition in muro of weakly esterified pectin [15], of callose, or of lignin at ectopic sites [16].

Genetic screening based on phenotypes has led to the identification of several additional genes [17-19] encoding KORRIGAN (KOR) and the cell surface proteins KOBITO (KOB) and COBRA (COB), mutations in which lead to cellulose deficiencies (kor, cob) and misorientation of cellulose microfibrils $(k o b)$.

A necessary requirement for cellulose biosynthesis is the supply of the UDP-glucose substrate. Among enzymes that might fulfil this role, sucrose synthase (SuSy) has received special attention because it produces UDP-glucose and fructose from sucrose, and has been shown to be tightly associated with the deposition of cellulose in cotton fibers [20-22]. However, a clear picture of its involvement awaits further investigation [23].

90 As a major structural component of the cell wall, cellulose has to be correctly orientated to 91 allow growth and optimal interactions with other polymers. A tight contact between the 92 cellulose synthase machinery and cytoskeleton dynamics has long been claimed [24]. Indeed, 93 confocal imaging has shown that isoxaben, an inhibitor of cellulose biosynthesis, alters the 94 organisation of cortical microtubules [25], and alters alignment of microtubules with the 95 cellulose synthases AtCESA7 and AtCESA6, tagged respectively with the green 96 (GFP:AtCESA7) and the yellow (YFP:AtCES6) fluorescent proteins [26,27]. Conversely,

97 marked changes in YFP-CESA6 organisation are observed when the microtubules (MT) are 98 disrupted using oryzalin. Even though the precise connection between cellulose synthase and 99 microtubules is not definitively established, it is clear that cellulose synthase and 100 microtubules do affect each other. 


\section{Dumas et al.}

101

102

103

104

\section{In muro cellulose-binding proteins}

Once in the wall, the cellulose microfibrils are bound to hemicelluloses through hydrogen bonds, thereby contributing to the architecture of the cell wall. The protein expansin can loosen this architecture, probably by weakening the non-covalent adhesion between these polysaccharides [28] without hydrolysing them. It has been shown that downregulation of one gene encoding an $\alpha$-expansin in Petunia reduces the amount of crystalline cellulose in the cell wall [29]. Cellulases (endo- $\beta$-1,4-glucanase, cellobiohydrolase, $\beta$-glucosidase) are another major category of plant and microbial enzymes that interact with cellulose, ultimately degrading cellulose to $\beta$-D-glucose. The presence of CBDs in some plant and microbial endocellulases participate in the efficacy of these enzymes by anchoring them to their substrate [30].

A search for microbial effectors has led to the isolation of a glycoprotein that is located in the inner and outer layers of the cell wall of Phytophthora parasitica, a pathogen of the tobacco plant Nicotiana tabacum [31]. Its protein moiety is composed of two direct cysteine-rich repeats connected by a linker. Each repeat contains a motif that closely resembles the fungal type I CBD consensus pattern found in cellulases from various fungi [32,33]. Further characterization showed that this molecule is able to bind to crystalline cellulose and to tobacco cell walls but does not possess any detectable hydrolytic activity on various polysaccharides [32]. Due to its elicitor effect and lectin-like activity, the molecule was named CBEL (for cellulose-binding elicitor lectin). CBEL is widespread in the genus Phytophthora [34]. In Phytophthora, it serves to organise polysaccharide ( $\beta$-glucan) deposition in the cell wall, and it allows adhesion of the mycelium to cellulosic substrates [11]. 


\section{Dumas et al.}

CBDs are found in proteins of microbial (bacteria, fungi, oomycetes) and plant (endocellulases, expansin) origins [35,36], and are also encountered in non-enzymic effectors secreted by nematode pathogens during plant colonisation [37]. Although exhibiting different sizes depending on their origins, they share common structural features and aromatic aminoacid residues implicated in cellulose binding affinity $[35,36]$. The aromatic residues of the two CBDs of CBEL predicted to be surface-exposed and involved in cellulose binding were deduced from sequence alignment with the cellobiohydrolase I CBD from the fungus Trichoderma reesei. CBDs are thought to locally disrupt hydrogen bonding between cellulose chains, resulting in local destabilization of cellulose microfibrils $[35,36]$.

Proteomic studies have shown that plant cell walls contain numerous proteins. A comparison of the patterns of wild-type and cellulose mutants might prove useful to uncover additional cellulose-interacting proteins.

\section{Cellulose and associated defense sensing}

Recent studies provide evidence that alteration of cellulose integrity is a warning signal to which the plant cell responds by activating defense pathways.

\section{Cellulose synthase-associated sensing}

Cellulose is the main load-bearing polymer of the plant cell wall to which it notably confers mechanical strength. Therefore, cellulose-deficient plants would be expected to be less resistant to pathogen ingress and abiotic stress. There are now several examples indicating that this is not the case.

The first contribution to this subject came from the report that a mutant (cev) of Arabidopsis showing constitutive expression of defense-related genes was mutated in the cellulose synthase gene CESA3 [38]. Interestingly this mutant showed increased production of the 


\section{Dumas et al.}

defense-related jasmonate (JA) and ethylene (ET) signalling molecules, and enhanced resistance to powdery mildew fungal diseases caused by Erysiphe cichoracearum, Erysiphe oronti, and Ö̈dium lycopersicum. A clear link between cell wall alteration and signalling via JA and ET was supported by the observation that chemical inhibition of cellulose biosynthesis could reproduce the phenotype on wild-type plants, and that this_phenotype i.e. cev was suppressed by mutations that interrupt the JA and ET pathways [39]. The notion that mutations of CESA3 activate defense responses through JA and ET was soon confirmed independently [16] on the ectopic lignin mutant (eli) of Arabidopsis. In this case, the decrease in cellulose content was compensated by an activation of lignin biosynthesis, thereby indicating that cellulose perturbation has many cellular effects.

Mutations in cellulose synthase genes involved in secondary cell wall formation are further examples linking cellulose deficiencies to biotic and abiotic stresses. Thus, the lew 2 mutant alleles of $A t C E S A 8$ are more tolerant to drought and osmotic stress than wild-type plants, possibly as a result of the accumulation of soluble sugars, proline and abscisic acid (ABA) within the cell following inhibition of cellulose synthesis [40]. A role for ABA in signalling was later illustrated in Arabidopsis mutants of the three CESA4, CESA7 and CESA8 subunits. These mutants had reduced levels of secondary cell wall cellulose, but showed enhanced resistance to three fungal species (Plectosphaerella cucumerina, Botrytis cinerea, Erysiphe cichoracearum), and to the vascular bacterial pathogen Ralstonia solanacearum [41]. In-depth genetic and transcriptomic studies revealed that signalling was independent of JA, ET and SA (salicylic acid), and correlated with ABA-induced defense, particularly against Ralstonia.

In response to various stresses, plants synthesize and accumulate callose, a $\beta$-1,3-glucan polymer, in their cell walls. The pmr4 mutant of callose synthase, another plasma membrane glucan synthase, was more resistant to the biotrophic pathogens Erysiphe cichoracearum, 


\section{Dumas et al.}

Erysiphe oronti, and Peronospora parasitica, an effect mediated by SA [42]. Since callose deposition normally participates in plant defense, this suggests that either deprivation of callose, or/and impairment of PMR4 behave as warning signals.

The above examples strongly support that defects in cell wall integrity are perceived by a surveillance system, which likely relies on a variety of sensor and receptor molecules.

\section{Cellulose Binding domains (CBDs) as emerging partners}

While studies of cellulose mutants were very fruitful in investigating the link between cellulose and defense responses, another approach could be the use of peptides or proteins with a disruptive activity on cellulose.

CBEL is a potent elicitor in the Phytophthora host plant tobacco, in which it induces local hypersensitive-like lesions, defense responses, and protection against subsequent infection with the oomycete [32]. It is also active on various non-host plants, notably on Arabidopsis. Using Arabidopsis mutants affected in the salicylic acid (SA), jasmonic acid (JA), and ethylene pathways (ET), it was shown that all three pathways are triggered by the elicitor. JA and ET are required for lesion formation, whereas induction of cell wall-associated defense proteins depends on SA [43]. In planta delivery of truncated or CBD-mutated versions of CBEL revealed that intact CBDs are essential for full elicitor and cellulose-binding activities [10]. Indeed, synthetic peptides corresponding to either CBD were sufficient to induce gene expression in tobacco, and to induce expression of a $G U S$ reporter gene under control of the defense $P R 1$ gene promoter in transgenic $P R 1:: G U S$ Arabidopsis. Interestingly, these CBDs did not provoke lesions following infiltration of tobacco and Arabidopsis. The fast (sec-min) and transient increase in cytosolic calcium that was observed upon incubation of tobaccoaequorin cells with CBEL required intact CBDs, and the presence of the cell wall. Whether other fungal type $1 \mathrm{CBDs}$ are able to induce plant defense awaits further investigations. One 


\section{Dumas et al.}

200

201

202

such example might be provided by the cellulase of Trichoderma viride whose membrane depolarization and other defense-related signalling effects are retained by the heat denaturated enzyme [44].

CBDs belong to a large superfamily of carbohydrate binding modules (CBMs) classified into more 40 different families, based on amino acid sequences, binding specificity, and structure. This large resource offers the opportunity to investigate precisely the cell wall sensing machinery since several of these CBMs specifically bind various cell wall components.

\section{Cellulose and cell wall sensing: a proposed model}

The above literature suggests that perturbation of the cellulose status, whether by mutation (cesA), adhesion (CBD), or chemical treatment (inhibitors), is a warning signal for the plant cell and leads to defense responses.

Is there a surveillance system common to such different stimuli? What do we know about the underlying mechanisms? A possible explanation is that they are perceived as a kind of mechanical stress. A similar situation is best documented in yeast, in which agents that cause cell wall stress, as well as mutations that impair cell wall biosynthesis, coactivate cell wall integrity (CWI) signalling and calcium signalling pathways [45], ultimately leading to cell wall biogenesis responses. Compared to yeast, what do we know of the mechanisms underlying cell wall sensing in plants? Could examining the responses induced by CBEL and CBDs provide answers?

In a proposed scenario, calcium would play a central role (Figure 1). Indeed the earliest effect related to cellulose perturbation recorded so far is a very fast increase (within the first minute) in cytosolic calcium in response to CBEL [10]. Interestingly, a plasma membrane protein named Mcal that plays a crucial role in mechano-stimulated $\mathrm{Ca}^{2+}$ uptake was recently 


\section{Dumas et al.}

characterized in Arabidopsis [46]. Another plasma membrane protein called THESEUS1 (THE1) that partly mediates growth inhibitory and ectopic lignin deposition phenotypes of the prc1-1 mutant of CESA6 of Arabidopsis [47] was also reported. THE1 is a receptor-like kinase which autophosphorylates, and requires a mutant ces $A 6$ background for its activation, suggesting that THE1 is involved in cellulose-sensing.

Knowledge of Mca1 now affords the possibility to check whether it mediates calcium entry in cellulose-deficient mutants and in response to CBEL and CBDs. In particular, does it interact with CESA and THE1? Whatever the case, increased cytosolic calcium might regulate phosphorylation processes, notably that of the THE1 and CESA proteins, leading to changes in their activity and, consequently, in the activity of their cell partners. One likely partner of CESA is SuSy, sucrose synthase, which catalyses the formation of UDPglucose, the substrate of CESA. Changes in its activity, i.e. glucose formation and/or sucrose consumption, might be perceived by a sugar-sensing machinery [48]. It was recently reported that sucrose can mediate priming of plant defense responses, and bring about broad-spectrum disease resistance in rice [49]. In the proposed model, a functional link between the CESA complex and the cytoskeleton MT might also be altered, as a result of the known depolymerisation of MT in the presence of increased calcium. MT depolymerisation has long been associated with defense responses against microbial invaders [50].

Additionally, or alternatively, the kinase activity of THE1 might be the starting point of a phosphorylation cascade leading to defense activation. Crosstalk between such a cascade and the JA, ET, SA, and ABA signalling pathways that are activated in response to cellulose perturbations can be predicted.

Based on this model, several questions emerge (Box 1). In particular, it will be interesting to know whether cellulose can relay information from external stimuli such as CBDs to intracellular signalling via cellulose synthase. The availability of Arabidopsis mutants and of 


\section{Dumas et al.}

250 CBDs together with cell biology imaging technology provides us with powerful tools to probe 251 the proposed model.

\section{Conclusion}

254 Although the notion of cell wall integrity-sensing is not novel, the underlying mechanisms are 255 still largely unknown. The recent findings that cellulose alterations are perceived as warning 256 signals in Arabidopsis provide us with a powerful model system to study the surveillance 257 system of the cell. Future work will aim at identifying novel genes involved in cell wall 258 sensing. This might be accomplished by screening mutants for their response to CBD 259 peptides. Understanding responses to cell wall damages might contribute to improve the 260 resistance of plants to biotic stresses.

261

262

264 Box 1. Outstanding questions

265 - How general among known CBDs is the effect induced by the CBDs of CBEL?

266 - Besides cellulose, do the CBDs interact with other cell-surface components such as the 267 wall-associated kinases (WAKs) or other protein kinases?

268 - Is the cellulose-CBD interaction perceived directly by a Mca1-like protein, or through the 269 cellulose-synthesizing machinery?

270 - Does the chemical inhibition of cellulose synthase induce $\mathrm{Ca}^{2+}$ changes and associated $271 \quad$ signalling events in a way similar to the CBDs?

272 - Can sucrose sensing and a CESA-MT link be involved in CBD-induced defense? 


\section{Dumas et al.}

273

274

275

276

277

278

279

280

281

282

283

284

\section{References}

1 Lamport, D.T.A. and Northcote, D.H. (1960) Hydroxyproline in primary cell walls of higher plants. Nature 188, 665-666

2 Darvill, A. and Albersheim, P. (1984) Phytoalexins and their elicitors: A defense against microbial infection in plants. Annu. Rev. Plant Physiol. Plant Mol. Biol. 35, 243-275

3 Esquerré-Tugayé, M.T. (2006) Hydroxyproline-rich glycoproteins in plant-microbe interactions. In The science and lore of the plant cell wall (Hayashi, T., ed.), pp. 303309, Brown Walker Press

$4 \quad$ von Röpenack, E. et al. (1998) Structural analyses and dynamics of soluble and cell wall-bound phenolics in a broad spectrum resistance to the powdery mildew fungus in barley. J. Biol. Chem. 273, 9013-9022

5 Humphrey, T.V. et al. (2007) Sentinels at the wall: cell wall receptors and sensors. New Phytol. 176, 7-21

6 Somerville, C. (2006) Cellulose synthesis in higher plants. Annu. Rev. Cell Dev. Biol. $22,53-78$

7 Joshi, C.P. and Mansfield, S.D. (2007) The cellulose paradox--simple molecule, complex biosynthesis. Curr. Opin. Plant Biol. 10, 220-226

8 Vorwerk, S. et al. (2004) The role of plant cell wall polysaccharide composition in disease resistance. Trends Plant Sci. 9, 203-209

9 Huckelhoven, R. (2007) Cell wall-associated mechanisms of disease resistance and susceptibility. Annu. Rev. Phytopathol. 45, 101-127

10 Gaulin, E. et al. (2006) Cellulose binding domains of a Phytophthora cell wall protein are novel pathogen-associated molecular patterns. Plant Cell 18, 1766-1777 


\section{Dumas et al.}

29811 Gaulin, E. et al. (2002) The CBEL glycoprotein of Phytophthora parasitica var-

299 nicotianae is involved in cell wall deposition and adhesion to cellulosic substrates. $J$.

$300 \quad$ Cell Sci. $115,4565-4575$

30112 Pear, J.R. et al. (1996) Higher plants contain homologs of the bacterial celA genes 302 encoding the catalytic subunit of cellulose synthase. Proc. Natl. Acad. Sci. USA. 93, $303 \quad 12637-12642$

30413 Persson, S. et al. (2007) Genetic evidence for three unique components in primary 305 cell-wall cellulose synthase complexes in Arabidopsis. Proc. Natl. Acad. Sci. USA 104 (39), 15566-15571

30714 Desprez, T. et al. (2007) Organization of cellulose synthase complexes involved in 308 primary cell wall synthesis in Arabidopsis thaliana. Proc. Natl. Acad. Sci. USA 104 309 (39), 15572-15577

31015 Burton, R.A. et al. (2000) Virus-induced silencing of a plant cellulose synthase gene. Plant Cell 12, 691-706

Cano-Delgado, A. et al. (2003) Reduced cellulose synthesis invokes lignification and defense responses in Arabidopsis thaliana. Plant J. 34, 351-362

31417 His, I. et al. (2001) Altered pectin composition in primary cell walls of korrigan, a 315 dwarf mutant of Arabidopsis deficient in a membrane-bound endo-1,4 $\beta$-glucanase. Planta 212, 348-358

Pagant, S. et al. (2002) KOBITO1 encodes a novel plasma membrane protein necessary for normal synthesis of cellulose during cell expansion in Arabidopsis. Plant Cell 14, 2001-2013

32019 Roudier, F. et al. (2002) The COBRA family of putative GPI-anchored proteins in Arabidopsis. A new fellowship in expansion. Plant Physiol. 130, 538-548 


\section{Dumas et al.}

$322 \quad 20$

323

324

$325 \quad 21$

326

327

328

329

330

331

332

333

$334 \quad 25$

335

336

337

338

339

Amor, Y. et al. (1995) A membrane-associated form of sucrose synthase and its potential role in synthesis of cellulose and callose in plants. Proc. Natl. Acad. Sci. USA. 92, 9353-9357

1 Haigler, C.H. et al. (2001) Carbon partitioning to cellulose synthesis. Plant Mol. Biol. $47,29-51$

22 Ruan, Y.L. et al. (2003) Suppression of sucrose synthase gene expression represses cotton fiber cell initiation, elongation, and seed development. Plant Cell 15, 952-964

23 Coleman, H.D. et al. (2006) Up-regulation of sucrose synthase and UDP-glucose pyrophosphorylase impacts plant growth and metabolism. Plant Biotechnol. J. 4, 87101

24 Baskin, T.I. (2001) On the alignment of cellulose microfibrils by cortical microtubules: a review and a model. Protoplasma 215, 150-171

Fisher, D.B. and Cyr, R.J. (1998) Extending the microtubule/microfibril paradigm. Cellulose synthesis is required for normal cortical microtubule alignment in elongating cells. Plant Physiol. 116, 1043-1051

26 Gardiner, J.C. et al. (2003) Control of cellulose synthase complex localization in developing xylem. Plant Cell 15, 1740-1748

27 Paredez, A.R. et al. (2006) Visualization of cellulose synthase demonstrates functional association with microtubules. Science 312, 1491-1495

Cosgrove, D.J. et al. (2002) The growing world of expansins. Plant Cell Physiol. 43, $1436-1444$

29 Zenoni, S. et al. (2004) Downregulation of the Petunia hybrida $\alpha$-expansin gene PhEXP1 reduces the amount of crystalline cellulose in cell walls and leads to phenotypic changes in petal limbs. Plant Cell 16, 295-308 


\section{Dumas et al.}

34630 Levy, I. et al. (2002) Modification of polysaccharides and plant cell wall by endo-1,4-

$347 \quad \beta$-glucanase and cellulose-binding domains. Biomol Eng. 19, 17-30

34831 Séjalon-Delmas, N. et al. (1997) Purification, elicitor activity, and cell wall

349 localization of a glycoprotein from Phytophthora parasitica var. nicotianae, a fungal

$350 \quad$ pathogen of tobacco. Phytopathology 87, 899-909

35132 Villalba-Mateos, F. et al. (1997) Cloning and characterization of a cDNA encoding an

352 elicitor of Phytophthora parasitica var. nicotianae that shows cellulose-binding and

353 lectin-like activities. Mol. Plant-Microbe Interact. 10, 1045-1053

35433 Gilkes, N.R. et al. (1991) Domains in microbial $\beta$-1,4-glycanases: sequence

355 conservation, function, and enzyme families. Microbiol. Rev. 55, 303-315

35634 Judelson, H.S. (2007) Genomics of the plant pathogenic oomycete Phytophthora:

357 insights into biology and evolution. Adv. Genet. 57, 97-141

35835 Hashimoto, H. (2006) Recent structural studies of carbohydrate-binding modules. Cell

$359 \quad$ Mol Life Sci 63 (24), 2954-2967

36036 Shoseyov, O. et al. (2006) Carbohydrate binding modules: biochemical properties and

361 novel applications. Microbiol. Mol. Biol. Rev. 70, 283-295

36237 Ding, X. et al. (1998) A secretory cellulose-binding protein cDNA cloned from the 363 root-knot nematode (Meloidogyne incognita). Mol. Plant-Microbe Interact. 11, 952364959

36538 Ellis, C. and Turner, J.G. (2001) The Arabidopsis mutant cev1 has constitutively 366 active jasmonate and ethylene signal pathways and enhanced resistance to pathogens.

$367 \quad$ Plant Cell 13, 1025-1033

36839 Ellis, C. et al. (2002) The Arabidopsis mutant cevl links cell wall signalling to 369 jasmonate and ethylene responses. Plant Cell 14, 1557-1566 


\section{Dumas et al.}

$370 \quad 40$

371

372
Chen, Z. et al. (2005) Disruption of the cellulose synthase gene, AtCesA8/IRXI, enhances drought and osmotic stress tolerance in Arabidopsis. Plant J. 43, 273-283

41 Hernandez-Blanco, C. et al. (2007) Impairment of cellulose synthases required for Arabidopsis secondary cell wall formation enhances disease resistance. Plant Cell 19, $890-903$

42 Nishimura, M.T. et al. (2003) Loss of a callose synthase results in salicylic aciddependent disease resistance. Science 301, 969-972

43 Khatib, M. et al. (2004) The CBEL elicitor of Phytophthora parasitica var. nicotianae activates defence in Arabidopsis thaliana via three different signalling pathways. New Phytol. 162, 501-510

44 Carden, D.E. and Felle, H.H. (2003) The mode of action of cell wall-degrading enzymes and their interference with Nod factor signalling in Medicago sativa root hairs. Planta 216, 993-1002

45 Levin, D.E. (2005) Cell wall integrity signaling in Saccharomyces cerevisiae. Microbiol. Mol. Biol. Rev. 69 (2), 262-291

46 Nakagawa, Y. et al. (2007) Arabidopsis plasma membrane protein crucial for $\mathrm{Ca}^{2+}$ influx and touch sensing in roots. Proc. Natl. Acad. Sci. USA 104, 3639-3644

47 Hematy, K. et al. (2007) A receptor-like kinase mediates the response of Arabidopsis cells to the inhibition of cellulose synthesis. Curr. Biol. 17, 922-931

48 Rolland, F. et al. (2006) Sugar sensing and signaling in plants: conserved and novel mechanisms. Annu. Rev. Plant Biol. 57, 675-709

49 Gomez-Ariza, J. et al. (2007) Sucrose-mediated priming of plant defense responses and broad-spectrum disease resistance by overexpression of the maize pathogenesisrelated PRms protein in rice plants. Mol. Plant-Microbe Interact. 20, 832-842 


\section{Dumas et al.}

39450 Gross, P. et al. (1993) Translocation of cytoplasm and nucleus to fungal penetration sites is associated with depolymerization of microtubules and defence gene activation in infected, cultured parsley cells. EMBO J. 12, 1735-1744 


\section{Dumas et al.}

397

398

399 Figure 1. A model for signal transduction linking CBD perception and cellulose alterations to 400 defense activation. (i) It is proposed that the fast increase in the cytosolic calcium induced by 401 CBEL treatment is mediated by a permeable mechanosensing channel system that relies on 402 Mcal or on a Mca1-like protein. (ii) Activation of Mcal would either occur directly in 403

\section{Legend to figure 1}

response to a changing physicochemical environment of the cell wall or through its interaction with a cellulose synthase-integrity sensor system (CESA, THE1). Mca1 and THE1 are two plasma membrane proteins related to $\mathrm{Ca}^{2+}$ influx (Mca1) and to cell wall integrity sensing (THE1) in Arabidopsis. (iii) Calcium might then activate phosphorylation processes, notably on the phosphorylable sites of CESA and THE1. (iv) Changes in CESA and THE1 activities would finally be the starting points of downstream signalling cascades leading to defense gene expression. Possible crosstalks with ethylene (ET), jasmonic acid (JA), salicylic acid (SA) and abscisic acid (ABA) signalling pathways that are induced by cellulose alterations are indicated. 


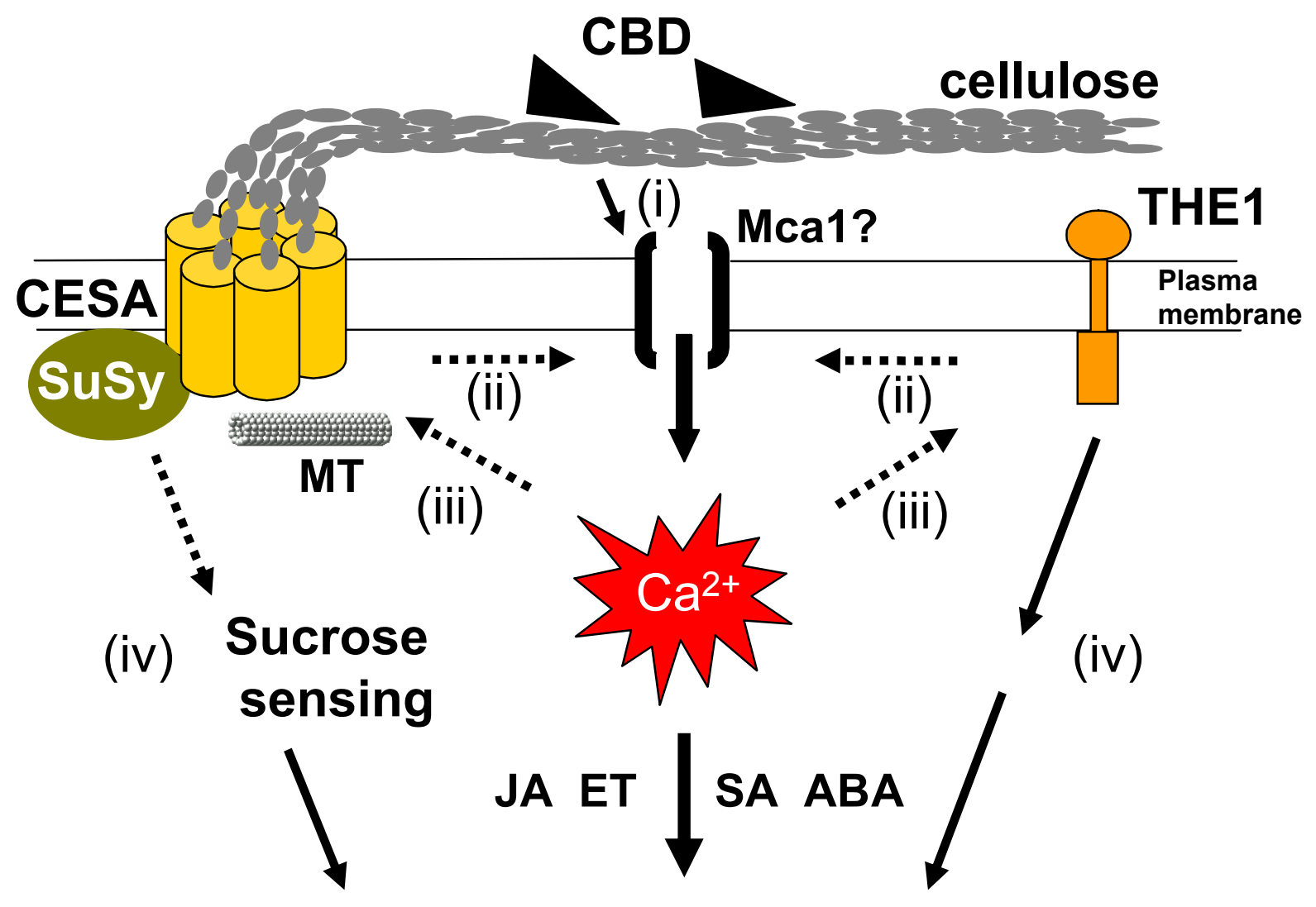

Defense gene expression

FIGURE 1 